\title{
Acquisition of the hierarchy of tonal functions in music
}

\author{
CAROL L. KRUMHANSL and FRANK C. KEIL \\ Cornell University, Ithaca, New York 14853
}

\begin{abstract}
The acquisition of the hierarchy of tonal stabilities in music is investigated in children of elementary school age. Listeners judge how good short tone sequences sound as melodies. The ratings show a pattern of increasing differentiation of the pitches in an octave range. The youngest listeners distinguish between scale and nonscale tones; older listeners distinguish between the tonic triad tones and other scale components. A group of adult listeners show octave equivalence and temporal asymmetries, with a preference for sequences ending on the more stable tones within the hierarchy. Pitch height effects do not interact with the age of the listener. These results are discussed in terms of the primacy of physical variables, noviceexpert differences, and general cognitive principles governing the acquisition and development of internal representations of pitch relationships.
\end{abstract}

Considerable attention in recent cognitive development research has been directed at determining how the structure of internal representations develops and changes with age. Domains of inquiry have included cognitive maps (Kosslyn, Pick, \& Fariello, 1974), stories (Mandler \& Deforest, 1979), scripts (Nelson \& Gruendel, 1981), and semantic knowledge (Keil, 1979). Little is known, however, about the development of the cognitive representation of musical structures. Developmental research on music perception has focused primarily on sensitivity to changes in more physically defined dimensions, such as the temporal reordering of tones, transpositions, and contour changes. These studies have not, for the most part, been concerned with describing the perception of musical relationships other than those that can be characterized directly in terms of pitch frequencies and changes in pitch frequencies in the externally presented stimulus.

There is now substantial evidence that music perception in adults is mediated through various cognitive operations that make reference to a system of knowledge about how pitches are typically used in combination in tonal music. This system of knowledge includes the distinctive interval relationships of the diatonic scale (Dewar, Cuddy, \& Mewhort, 1977; Dowling, 1978), the tonal functions of single pitches (Krumhansl, 1979; Krumhansl \& Kessler, 1982; Krumhansl \&

This research was supported by grants from the National Science Foundation to Frank C. Keil (BNS-78-06200) and to Carol L. Krumhansl (BNS-81-03570). The authors are grateful to Richard Tocco of the Ithaca Public School District for his kind cooperation. The authors thank Lionel Wolberger, who assisted in designing and conducting the experimental sessions, and Kim Pompey, who also helped with the data collection. Reprint requests may be addressed to either author: Department of Psychology, Uris Hall, Cornell University, Ithaca, New York 14853.
Shepard, 1979) and chords (Bharucha \& Krumhansl, in press; Krumhansl, Bharucha, \& Kessler, 1982) in musical keys, and the distances between musical keys (Bartlett \& Dowling, 1980; Cuddy, Cohen, \& Miller, 1979; Krumhansl \& Kessler, 1982; Krumhansl, Bharucha, \& Castellano, in press). Empirical support for these internalized properties comes from direct judgments of combinations of musical tones and chords and from memory performance. Through experience with well-structured musical sequences, listeners apparently have identified certain underlying regularities that by their nature must be encoded in a form more abstract than the pitch frequencies of the musical stimulus. We refer to this knowledge as the internal representation of tonal pitch structure.

Krumhansl and Kessler (1982) have argued that a basic component of this internal representation is a hierarchy of stabilities within the set of musical tones and that structure at the level of chords and musical keys can be seen to a great extent as derivative of this hierarchy. Reference to this internalized hierarchy of tonal stability, which identifies certain tones as most structurally central to the tonal system, is presumed to be essential to the apprehension of musical organization. Two empirical studies (Krumhansl \& Kessler, 1982; Krumhans1 \& Shepard, 1979) have provided quantitative description of this internalized tonal hierarchy, and its acquisition in children of elementary school age is investigated in the present study.

Our focus on the tonal hierarchy is based on musictheoretic accounts of musical structure. These descriptions, which are derived from analyses of musical compositions, suggest that organization in tonal music can best be understood in terms of the sequential combinations of pitches that vary in terms of stability within the tonal framework (Meyer, 1956). In Western music, the musical tones consist of a discrete set of 
Table 1

Musical Elements in Key of C,Major

\begin{tabular}{|c|c|c|c|c|c|c|c|c|c|c|c|c|c|}
\hline & & & & Ausica & me & $\mathbf{n} \mathbf{K}$ & $I C, M$ & & & & & & \\
\hline $\begin{array}{l}\text { Chromatic Scale } \\
\text { Diatonic Scale } \\
\text { Tonic Triad } \\
\text { Tonic Tone }\end{array}$ & $\begin{array}{l}\mathrm{C} \\
\mathrm{C} \\
\mathrm{C} \\
\mathrm{C}\end{array}$ & $\mathrm{C \#}$ & $\begin{array}{l}\mathrm{D} \\
\mathrm{D}\end{array}$ & D\# & $\begin{array}{l}\mathrm{E} \\
\mathrm{E} \\
\mathrm{E}\end{array}$ & $\begin{array}{l}F \\
F\end{array}$ & $\mathrm{~F} \#$ & $\begin{array}{l}\mathrm{G} \\
\mathrm{G} \\
\mathrm{G}\end{array}$ & $\mathrm{G} \#$ & $\begin{array}{l}\mathrm{A} \\
\mathrm{A}\end{array}$ & $\mathrm{A \#}$ & $\begin{array}{l}\text { B } \\
\text { B }\end{array}$ & $\begin{array}{l}C^{\prime} \\
C^{\prime} \\
C^{\prime} \\
C^{\prime}\end{array}$ \\
\hline
\end{tabular}

pitches from the frequency continuum that are equally spaced in terms of log frequency with 12 pitches/octave. The pitches in the octave range from middle $\mathrm{C}$ on the piano to the $\mathrm{C}$ an octave above (denoted $\mathrm{C}^{\prime}$ ) are shown in Table 1. However, the pitches that are selected for a composition are typically those that conform to the intervallic structure of the diatonic scale; those in the $\mathrm{C}$ major scale are noted in Table 1 . This subset contains a distinctive pattern of half and whole steps that uniquely defines the musical key. Certain of these elements, particularly the first step of the scale (the tonic) and, to a lesser extent, the fifth and third scale steps, are described by music theorists as more central, stable tones. In the key of $\mathrm{C}$ major, these are $\mathrm{C}$ (or $\mathrm{C}^{\prime}$ ), $\mathrm{E}$, and $\mathrm{G}$, which together form the major tonic chord. These particular tones tend to appear in more prominent positions, such as at the beginning and end of a melody and in rhythmically stressed positions, and they are sounded more frequently than other tones. Among these, one particular tone called the tonic, $\mathrm{C}$ (and its octave equivalents) in the case of the $\mathrm{C}$ major key, is the most stable tone within the system. This tone serves as a central reference point to which all other tones have a well-defined relationship. According to this account, melodic structure depends on the emphasis given to particular tones within a set of elements that contains a characteristic pattern of frequency ratios.

This description of musical structure has been shown to apply to the psychological process of music perception in listeners familiar with tonal music. Krumhansl and Shepard (1979) presented listeners the first seven notes of an (incomplete) major scale followed by any one of the single pitches in the next octave range. Listeners rated how well the final tone completed the sequence. The most musically trained listeners gave a distinctive pattern of ratings, with the tonic tones preferred to the other scale tones and scale tones preferred to nonscale (nondiatonic) tones. The fifth and third scale degrees were preferred somewhat to the remaining nontonic scale steps. This pattern has been replicated in a more recent study (Krumhansl \& Kessler, 1982) using a variety of different key-defining elements, such as scales, chords, and cadences (chord sequences that are traditionally used to indicate key and to signal phrase endings). The rating profiles serve as a distinctive marker of tonal organization and, when shifted to different tonics and intercorrelated, produced a regular and interpretable spatial map of the musical keys.
A scaling study of pairs of tones presented in a major scale or chord context also recovered the tonal hierarchy (Krumhansl, 1979). Multidimensional scaling of the judgments produced a conical structure in which the tones were ordered around the surface of the cone according to pitch height so that the two tonics separated by an octave were located in proximal positions. The components of the tonic triad chord formed a closely interrelated subset near the vertex of the cone, the remaining scale tones were more widely separated on a ring farther from the vertex, and the nondiatonic tones were located farthest from the vertex, being only distantly related to the diatonic tones and to each other. Thus, the hierarchy of stabilities was represented in the multidimensional scaling solution as distance from the vertex. Temporal asymmetries were also found in the ratings of pairs of tones, such that pairs ending on more stable tones received higher ratings than those that moved from a more stable to a less stable tone.

In two recognition memory experiments, Krumhansl (1979) also found that nondiatonic tones were more difficult to remember than diatonic tones in a tonal context and were more frequently confused with diatonic tones than the reverse. Finally, Cuddy et al. (1979) showed that changes to nondiatonic tones were easier to detect than changes within the diatonic scale, and Dowling's (1978) listeners had difficulty detecting changed intervals when the scale structure and pitch contour (the pattern of increasing and decreasing frequencies) were preserved. Thus, the hierarchy of stability, which distinguishes between diatonic and nondiatonic tones and further identifies particular elements within the scale as most central, is reflected in a variety of tasks. In this is seen the operation of what may be described as a perceptual schema (Neisser, 1976), which is used to encode the incoming musical tones and which gives rise to certain expectations about what tones will be sounded subsequently.

Given this evidence that adult listeners encode tonal material in terms of a hierarchy of stability that applies to the key of the broader context, the question arises as to how this aspect of musical knowledge develops. Dowling (in press-a) and Pick (1979) have recently provided excellent reviews of the available developmental literature on music perception, which will be summarized only briefly here. Young infants are able to match single pitches by actually producing them (Kessen, Levine, \& Wendrich, 1979) and show dishabituation to temporally scrambled versions of sequences of tones 
(Chang \& Trehub, 1977; Melson \& McCall, 1970). The Chang and Trehub study showed that dishabituation to the permuted version was larger than that to an exact transposition, which takes the sequence to a different pitch range but maintains both the relative sizes of the intervals and the contour (the pattern of ups and downs). The available studies do not demonstrate whether maintaining the interval sizes or simply the contour patterns is the basis of this result, but Dowling's work (Dowling, 1978; Dowling \& Fujitani, 1971) suggests that the contour possibility is more likely. Gardner, Davidson, and McKernon (1980) found that 2- and 3-year-old children reproduced the contours of short phrases, but with considerable variation in the pitches. Gardner et al. demonstrated that 4-year-old children could maintain scale steps within phrases, but they might shift to different keys at phrase boundaries; only 5 -year-olds maintained the key throughout extended melodies.

Other studies (Imberty, 1969; Zenatti, 1969; both cited in Dowling, in press-a) found a somewhat later age at which the scale structure becomes firmly estab. lished. Zenatti had children judge which note of a threenote sequence had been altered, and only by age 6 or 7 years did a difference in performance emerge between tonal and random sequences. By age 7 years, changes between keys in the middle of familiar tunes were detected in the study by Imberty. Finally, Dowling (in press-a) noted that by age 5 years children do better on recognition memory tasks with familiar materials than with novel, randomly generated sequences of tones. Thus, only by the age of elementary school do the pitches stabilize to conform to the scale structure, as evidenced by an increasing ability to accurately produce and recognize tone sequences, to maintain a key throughout a melody, and to notice shifts between keys. Differences in performance on tonal and random sequences, moreover, emerge during this age. Dowling (in press-a) characterizes the developmental trend found in the available literature as one in which more gross structural features are acquired earlier than more subtle aspects having to do with the structuring of pitches within the constraints of the Western diatonic scale.

The present investigation traces the acquisition of the internalized hierarchy of tonal stabilities. Children of elementary school age were asked to make ratings about how good short melodies sounded. Each melody began with four tones, the components of a major triad chord, with the tonic repeated in the first and third temporal positions. This choice was based on the conventional emphasis given to more stable tones of the key within well structured melodies. The final two tones were both drawn from the scale of the key established by the context (the key in which the major chord is the tonic triad) or were both nondiatonic tones. A group of adult listeners was also included, because the context in this study differed somewhat from that employed in the earlier scaling study of musical tones using adult listeners (Krumhansl, 1979), which had an otherwise similar design.

Research in other areas of cognitive development, as well as research on music itself, suggests that certain features might become perceptually salient before others. For example, one frequent theme in cognitive development research is that children are more sensitive to simple, physically defined properties of stimuli than to more abstract, higher order relationships. This theme can be seen in discussions of the development from iconic to symbolic representations (Bruner, Olver, Greenfield, et al., 1966), the shift from classifications based on perceptual attributes to classifications based on functional attributes (Bruner et al., 1966), and the development from concrete to abstract representations (e.g., Vygotsky, 1962; Werner \& Kaplan, 1963).

This sort of perspective would suggest that factors such as pitch height, repetitions of tones, and possibly octave equivalence would emerge relatively early in the present study compared with factors such as the distinction between diatonic and nondiatonic tones. This would be the case, since the former properties are represented relatively directly in the acoustic structure of the tones and may be encoded at early stages of sensory processing. If children do tend to progress from being most sensitive to basic physical information to more abstract, relational constructs, then this sort of progression would be expected.

A second view of cognitive development argues that children and adults differ primarily in terms of the size or complexity of their relevant knowledge bases, that is, children are simply novices in more domains than adults are (Chi, 1978). If one takes this view, the scale completion study (Krumhansl \& Shepard, 1979) makes certain predictions about the developmental sequence. In that study, different patterns in the ratings were found that depended on the extent of the listener's musical training. For those listeners with the least extensive musical backgrounds, the pitch height dimension predominated, and the two tonics separated by an octave were treated as partially equivalent. For listeners with a moderate level of training, the pitch height effect was greatly reduced, octave equivalence became more complete, the tonics received relatively high ratings, and there was a slight preference for other scale tones over nondiatonic tones. This preference for scale tones, particularly the components of the tonic triad chord, became more pronounced for the relatively expert listeners. For these listeners, octave equivalence was essentially complete and the pitch height dimension was absent from the pattern of responses. Thus, both the children-as-novices point of view and the position that simple physical characteristics are primary predict that pitch height and octave equivalence will be factors governing the responses of the youngest children. 
A different view of cognitive development (Gelman, 1978; Keil, 1981; Mandler, in press) argues that children and adults share throughout development certain predispositions to organize knowledge in specific ways. These predispositions are assumed to be essential for initially establishing and guiding the development of knowledge structures. Thus, underlying what may seem to be dramatic changes in cognitive development, there may be highly restrictive principles of organization, principles that hold cross-culturally. This general orientation suggests that cross-cultural considerations are relevant (Cole \& Means, 1981). Although the particular interval patterns of Western scale structure are unique to this musical culture, parallels exist in other musical traditions. Dowling (1979, in press-b) notes the universal selection of a subset of discrete scale pitches in all musical cultures, typically between five and seven in an octave range. Moreover, with few exceptions, the pitches are repeated at octave intervals and are often sounded in unison. There is a characteristic pattern of varied interval sizes within the octave range. Certain pitches tend to be emphasized in a particular musical context, and when additional pitches are included, they function as ornamentation for more central tones. Thus, structured sets of scale pitches, certain of which are emphasized within a particular context, and octave equivalence appear in most musical traditions. These parallels suggest that not only octave equivalence and pitch height, but also a hierarchy of stabilities within the set of musical tones, will be present in the developmental sequence from an early age.

In summary, the first two views of cognitive development predict a pattern of acquisition wherein subjects would first be more sensitive to aspects of the musical sequence that are more directly recoverable from a physical description of the acoustic stream. The third view, however, puts more emphasis on universal structural principles, and cross-cultural comparisons of musical traditions suggest that the hierarchy of tonal stabilities may be basic.

\section{METHOD}

\section{Subjects}

The 52 children who participated in the experiment were volunteers from music classes in the Ithaca public schools. Of the 14 first- and second-grade students, 6 had taken lessons on a musical instrument, with an average of .9 years instruction; the remaining subjects had had no music lessons. In all but one case, however, someone in the child's family played an instrument at home. Fourteen subjects were drawn from the third and fourth grades. Of these, 10 had taken lessons on an instrument for an average of 1.1 years. Additionally, all but one of their families included someone who played an instrument. The group of 14 fifth- and sixth-grade students had 11 members taking music lessons, averaging 1.4 years instruction, and all but 1 had some family member who played an instrument. In addition, all of the children participated in the music class in their school for either .5 or $1 \mathrm{~h} /$ week. Thus, although no attempt was made to select the subjects for musical background, the group of partici- pants would be described as having some experience with music, the older, children having naturally more musical background than the younger children.

The 14 adult subjects were undergraduate or graduate students at Cornell University who volunteered their time. Of these, 12 had taken lessons on a musical instrument for an average of 7.8 years, and all but 1 came from a household in which at least one other member played an instrument. Again, the selection of subjects was not made on the basis of musical background, but, on the average, quite extensive musical training was represented.

\section{Stimulus Materials}

The sequences were played on an electric piano and recorded on Maxell 46 cassette tapes. During the experimental sessions, they were played back on a Sanyo (TRC 1500) cassette tape recorder. Two different stimulus tapes were recorded, using different random orders of trials, and half the subjects within each group heard one of these tapes and half heard the other tape.

Each tape began with seven sample sequences to familiarize the listeners with the task and the general kind of sequences they would hear in the experiment. The first four sample trials had melodic sequences that were longer than those used on the experimental trials, so as to indicate that the later sequences were to be considered as the beginning of a longer melody. The last three sample sequences had the same structure as the experimental trials. Following this, there were six practice trials that were selected to be representative of the experimental trials. These were followed by the 63 trials of the experiment.

Each trial in the main experiment consisted of six tones. The first four tones, $C_{3} E_{3} C_{3} G_{3}$ (where the subscript indicates that the notes were sounded in the octave below middle $C, C_{4}$ ), had durations of approximately $.3 \mathrm{sec}$. These were followed immediately by two tones selected from the range $C_{4}$ to $C_{5}$ inclusive that sounded for approximately $.6 \mathrm{sec}$ each. All possible ordered pairs of tones in the $C$ major scale $\left(C_{4} D_{4} E_{4} F_{4} G_{4} A_{4} B_{4} C_{5}\right)$ were presented once, giving 56 combinations. In addition, there were seven trials that had two nonscale tones. They were: $A \#_{4} \mathrm{C}_{4}, \mathrm{C} \#_{4} \mathrm{G} \#_{4}, \mathrm{C} \#_{4} \mathrm{~A} \#_{4}, \mathrm{G} \#_{4} \mathrm{~F} \#_{4}, \mathrm{~F} \#_{4} \mathrm{G} \#_{4}, \mathrm{~A} \#_{4} \mathrm{C} \#_{4}$, and $\mathrm{G} \#_{4} \mathrm{C} \#_{4}$. These nondiatonic trials were distributed among the 56 diatonic trials.

The children made their responses by choosing one of seven white dots ordered along a black line. Above the leftmost dot was a frowning face, above the middle dot was a neutral face, and above the rightmost dot was a smiling face. The experimenter coded these responses on a 7-point scale. The adults wrote down their responses using a 7-point scale, where 1 was designated "Sounds bad" and 7 was designated "Sounds good."

\section{Procedure}

The subjects were told that the experimenter was trying to write a song and were asked to judge how good or bad each sequence sounded. They were encouraged to use the full range of the response scale and were told to consider the short sequences as the beginning of a melody. All listeners, who were seen individually, first heard and judged the sample sequences, followed by the practice trials, and then the 63 experimental trials. Most children elected to take a short break sometime near the middle of the experiment. The sessions lasted approximately 20 to $25 \mathrm{~min}$. After hearing all of the trials, the subjects were asked about their musical backgrounds. Three children who exhibited difficulty on the practice items were replaced by other children from the same age group.

\section{RESULTS}

Before performing any statistical analyses, each subject's ratings were normalized. That is, the average 
Table 2

Trial Types and Average Normalized Ratings

\begin{tabular}{lllrrrr}
\hline \multicolumn{1}{c}{ Condition } & First Tone & Second Tone & Grades 1-2 & Grades 3-4 & Grades 5-6 & Adults \\
\hline Tonic-Tonic & C C' & C C' & -.183 & .464 & .687 & 1.121 \\
Triad-Triad & C E G C' & C E G C' & .260 & .485 & .611 & .800 \\
Nontriad-Triad & D F A B & C E G C' & .095 & .100 & .156 & .340 \\
Triad-Nontriad & C E G C' & D F A B & .045 & .053 & .037 & -.107 \\
Nontriad-Nontriad & D F A B & D F A B & .017 & -.161 & -.263 & -.279 \\
Nondiatonic & & & -.669 & -.900 & -1.053 & -1.518 \\
\hline
\end{tabular}

response for a subject was subtracted from each rating and this difference was divided by the standard deviation of that subject's responses. This normalization was done because there were quite large individual differences in the use of the response scale and these differences related to the age of the subject such that younger subjects tended to give higher ratings. All of the analyses to be described were based on the normalized scores; analogous analyses on the raw scores showed qualitatively similar effects.

The two tones at the end of each melodic sequence were all possible pairs of tones within the $\mathrm{C}$ major diatonic scale in an octave range or two nondiatonic tones. These pairs of tones could be classified as falling into one of the following six categories: (1) two tonic tones ( $C, C^{\prime}$, where $C^{\prime}$ denotes the $C$ above middle $C$ ), (2) two tones contained in the tonic triad chord $\left(C, E, G, C^{\prime}\right)$ not both tonics, (3) the first tone in the diatonic scale but not in the major triad (D, F, A, B) and the second tone in the tonic triad $\left(C, E, G, C^{\prime}\right),(4)$ the first tone in the tonic triad $\left(C, E, G, C^{\prime}\right)$ and the second tone in the scale but not in the tonic triad (D, F, A, B), (5) two diatonic tones, neither of which was in the tonic chord (D, F, A, B), and (6) two nondiatonic tones. These trial types are shown in Table 2, together with the average normalized score for each group of subjects.

\section{Effects of Age on Each Type of Tone Pair}

A two-way analysis of variance on the six trial types and the four groups of subjects showed a significant effect of trial type $[F(5,260)=99.054, p<.001]$ and a significant interaction between trial type and age $[F(15,260)=7.218, p<.001]$. The effect of age on the average rating for each type of tone pair will be considered first.

Looking across the first three rows of Table 2 , an increase in the average rating in the first three conditions is apparent. The increase is particularly marked for the tonics separated by an octave $[F(3,52)=6.798$, $p<.001]$. The ratings for other pairs of tones within the major chord also increased with age $[F(3,52)=$ $5.866, \mathrm{p}<.01]$. The ratings of pairs beginning on a nontriad tone and ending on a tone in the major triad chord were also found to increase $[\mathrm{F}(3,52)=5.273$, $\mathrm{p}<.01]$. Thus, for all melodies ending on a tone within the tonic triad chord, there was a significant increase in the obtained ratings with age.
These increases were balanced by a tendency for the ratings of the last three types of tone pairs to decrease with age. In the ratings given the pairs that began on a component of the major triad and ended on a nontriad tone, there was a decreasing trend that approached significance $[F(3,52)=2.346, .05<\mathrm{p}<.10]$. The pairs containing two nontriad tones showed a stronger decline $[F(3,52)=4.072, p<.05]$, and there was a striking drop in the ratings of the nondiatonic tones $[\mathrm{F}(3,52)=$ $10.286, \mathrm{p}<.001]$. Thus, with age, there was an increasing differentiation between tone pairs of the first three types, which all ended on components of the major triad chord, and the last three types, which ended either on a nontriad scale tone or on two nondiatonic tones.

\section{Structure Within Each Age Group}

In order to explore the trial type effects for each age group, the results were analyzed separately for each group of subjects. A one-way analysis of variance performed on the six trial types for the subjects in the first and second grades gave a significant effect of the type of tone pair $[F(5,65)=5.006, p<.001]$. A planned comparison showed a significant difference between the nondiatonic pairs (Condition 6) and the diatonic pairs (Conditions $1,2,3,4$, and 5) $[\mathrm{F}(1,65)=$ $20.198, \mathrm{p}<.001]$. There were, however, no differences among the remaining conditions, as shown in a separate analysis of variance on the first five conditions $[F(4,52)$ $=1.208, \mathrm{p}>.30]$. There was a tendency for the tonics separated by an octave to receive lower ratings than the other pairs within the tonic triad chord $[F(1,65)=4.643$, $\mathrm{p}<.05]$, but this may be accounted for by the maximally large distance between the two tonic tones (see below). For the first- and second-grade subjects, then, there was a strong division between the diatonic and nondiatonic pairs, and no regular differentiation among the remaining conditions. This finding is summarized at the top of Figure 1, which shows the average ratings for the diatonic and nondiatonic trials. In order to determine whether there was additional structure lost by collapsing the responses into these six trial types, multidimensional scaling (Kruskal, 1964; Shepard, 1962a, 1962b) and hierarchical clustering (Johnson, 1967) techniques were applied to the ratings for all diatonic pairs. These analyses revealed no additional regular or interpretable structure. 


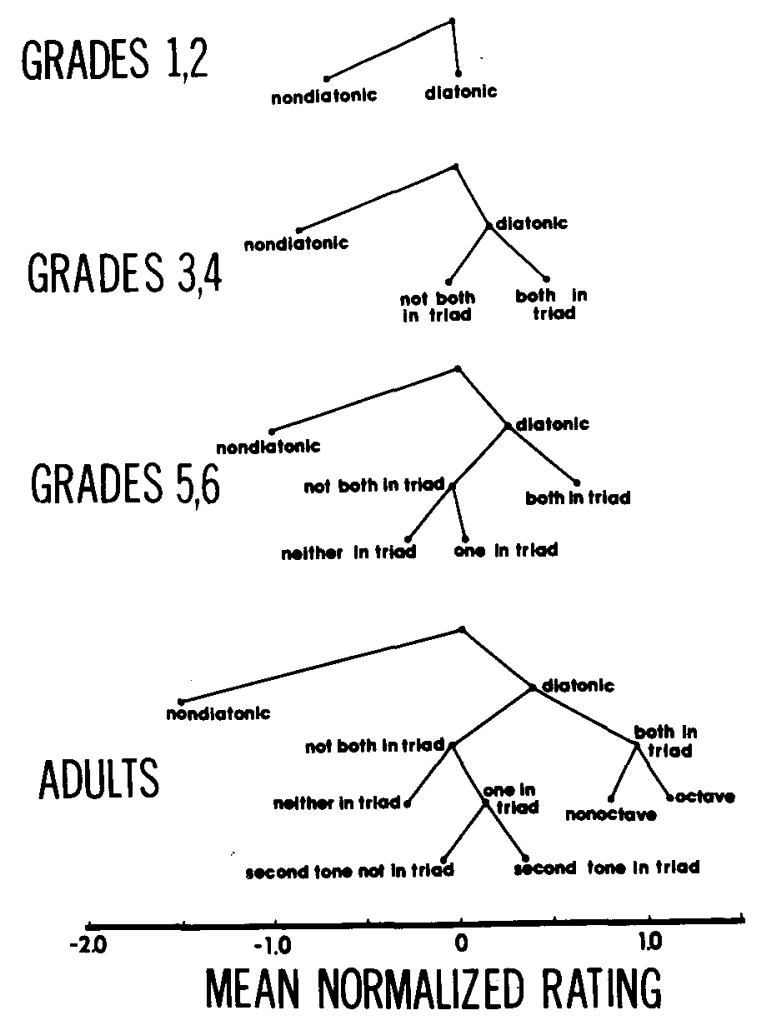

Figure 1. Significant differences found between trial types for the four groups of subjects: Grades 1 and 2, Grades 3 and 4, Grades 5 and 6, and adults.

In the responses for the third- and fourth-grade subjects, there was again a significant effect of trial type $[F(5,65)=10.843, p<.001]$, with an even stronger separation between diatonic and nondiatonic pairs $[F(1,65)=75.396, p<.001]$. In addition, there was a significant difference between pairs containing two tonic triad tones (Conditions 1 and 2) and conditions in which at least one tone was drawn from outside the tonic triad (Conditions 3, 4, and 5) $[F(1,65)=20.851$, $\mathrm{p}<.001]$ from a planned comparison. These results are summarized in the second panel of Figure 1. There were no differences within these two sets of conditions, except that trials containing two nontriad tones (Condition 5) tended to be rated lower than those containing one triad and one nontriad tone (Conditions 3 and 4) $[\mathrm{F}(1,65)=2.866, \quad .05<\mathrm{p}<.10]$. The scaling and clustering solutions showed a fairly compact cluster consisting of the $\mathrm{C}, \mathrm{E}, \mathrm{G}$, with the $\mathrm{C}^{\prime}$ relatively distant from the other triad tones.

The results for the fifth- and sixth-grade subjects contained a significant main effect of trial type $[\mathrm{F}(5,65)$ $=31.341, \mathrm{p}<.001]$. Again, a very large difference was found between diatonic and nondiatonic pairs $[F(1,65)$ $=107.771, p<.001]$. The difference between tonic triad pairs (Conditions 1 and 2) and other diatonic pairs (Conditions 3, 4, and 5) was also significant $[F(1,65)=41.583, p<.001]$. Moreover, these subjects gave lower ratings to pairs in which neither tone was in the tonic triad (Condition 5) than they gave to pairs with one triad and one nontriad tone (Conditions 3 and 4) $[F(1,65)=6.585, p<.05]$. These differences are depicted in the third panel of Figure 1. The difference between Conditions 3 and 4 approached significance $[F(1,13)=2.687, .10<p<.15]$, with a slight preference for sequences ending on a component of the tonic chord. However, no difference was found between the tonic pairs (Condition 1) and the other pairs in the tonic triad (Condition 2). The scaling and clustering analyses, which produced a quite closely interrelated group containing the $\mathrm{C}, \mathrm{E}, \mathrm{G}$, and $\mathrm{C}^{\prime}$, did not reveal any additional structure in the ratings of these subjects.

Finally, the ratings in the six conditions given by the adult subjects were analyzed. The main effect of condition in the analysis of variance was significant $[F(5,65)=$ $118.013, \mathrm{p}<.001]$. There was a large difference between the diatonic and nondiatonic pairs $[F(1,65)=401.821$, $\mathrm{p}<.001]$ and a preference for tone pairs within the tonic triad (Conditions 1 and 2) over the other diatonic pairs (Conditions 3, 4, and 5) $[F(1,65)=153.804$, $\mathrm{p}<.0011$. Also, as before, there was a preference for pairs with one triad and one nontriad tone (Conditions 3 and 4) over pairs with two nontriad tones (Condition 5) $[F(1,65)=14.010, p<.001]$. The data for the adults showed two additional effects. First, there was a preference for the tonic pairs (Condition 1) over the other pairs within the major triad (Condition 2) $[F(1,65)=$ $6.928, \mathrm{p}<.02$ ]. Second, within Conditions 3 and 4 , which contained one nontriad and one triad tone, there was a strong preference for sequences ending on a tone of the tonic triad $[F(1,65)=13.498, p<.001]$. These effects are summarized at the bottom of Figure 1, which also reflects the major patterns contained in the multidimensional scaling and clustering solutions.

\section{Pitch Height Effects}

In order to determine whether the difference in pitch of the two final tones affected the ratings, one final analysis was performed on the diatonic trials. For each subject, the average rating for pairs separated by a single scale step was computed; there were 14 such pairs. Next, the ratings for the 12 pairs separated by two scale steps were averaged. Average ratings were also found for the 10 pairs differing by three scale steps, the 8 pairs differing by four scale steps, and the 12 pairs differing by five or more scale steps. These values were analyzed in a two-way analysis of variance for the five distances and the four groups of subjects. This analysis showed a significant effect of pitch difference $[F(4,52)$ $=7.082, \mathrm{p}<.001]$, such that the ratings decreased as the distance between the two tones increased. There was no interaction between subject group and the distance between the two tones. Thus, all subjects gave higher ratings to pairs of tone separated by a smaller number of scale steps, and there was no developmental change in this effect. 


\section{DISCUSSION}

The present results showed an increasing differentiation among the musical tones as they function within the hierarchy of stabilities that applies to the key established by the melodic context. The first distinction to emerge was that between diatonic and nondiatonic pairs of tones. Higher ratings were given by the youngest children in Grades 1 and 2 to melodies ending on two scale tones than to those ending on nondiatonic tones. That is, the ratings reflected the internalization of the diatonic scale structure of Western music at a relatively early age.

The finding that the diatonic-nondiatonic distinction appears earlier than other factors in the present experiment is noteworthy. Because of the harmonics (overtones) of the tones used, the listeners might have been expected to have achieved octave equivalence of the two tonics at an earlier age than found here. Additionally, the context sequences, which explicitly contained the tones of the tonic triad chord, might have induced a preference for these tones over the other tones. Nonetheless, the repetition of the tonic triad tones in the final two positions did not produce higher ratings for these melodies. Moreover, pitch height was only a moderately strong factor and was not found to interact with age. The results do not support the prediction that simple physical properties of the sequences would predominate for the youngest listeners. Although these properties may well be perceived, they were not found to strongly influence the judgments of how good these short tone sequences sounded as melodies. Instead, the more abstract distinction between the tones contained in the scale (which were not all present in the context sequence) and the nonscale tones emerged first. Apparently, through experience with well structured tonal melodies, which contain overlapping subsets of the scale tones, even the youngest subjects had achieved a sense of which pitches do and do not belong within a tonal context. Dowling (1978, in press-b) has suggested the primacy of scale structure for processing melodies by adults.

By Grades 3 and 4, the responses showed a preference for melodies that ended on two tones of the tonic triad chord over other scale steps. These tones, the first (tonic), third, and fifth scale steps, as members of the tonic triad chord, are the most stable within the tonal system. Thus, the responses at this age began to reflect a differentiation among the set of scale tones induced by emphasizing certain tones within the context. This differentiation becomes more precise by Grades 5 and 6 , at which time there was a preference for sequences containing one, rather than no, tonic triad component in the final two positions. On trials with one triad and one nontriad tone, the oldest children showed a slight preference for sequences with the tonic chord in the final position. Apparently, temporal asymmetries involving tones of differential stability, which were quite strong in the ratings of the adult listeners in this and earlier studies (Krumhansl, 1979), appear to represent a rather late developmental phenomenon.

None of the groups of children in the experiment gave higher ratings to the tonics separated by an octave than to other pairs of tones in the tonic triad. Undoubtedly, this reflects the large pitch height difference between these tones. Indeed, small steps predominate in melodies (Jeffries, 1974; Ortmann, 1926), and a general effect of pitch height was found for all groups of subjects in this experiment. Only for the adult listeners did the strong relationship between the tonics separated by an octave prevail over the preference for small scale steps.

The observed developmental changes contrast with those found as a function of training in adults in the scale completion study (Krumhansl \& Shepard, 1979). Based on the earlier results, the following sequence of acquisition might have been anticipated: pitch height, octave equivalence, diatonic-nondiatonic, and tonic triad vs. other scale tones. In that study, the pitch height effect was virtually absent from the responses of the listeners with the most extensive musical backgrounds. The developmental sequence did not parallel these changes found as a function of training, despite the fact that the novice adults were roughly equivalent to many of the children, particularly the youngest children, in terms of formal music instruction. Possibly, this finding may be accounted for by different strategies that the children and adult listeners brought to the particular experimental task, or it may in fact reflect real differences in aptitude for assimilating abstract properties of musical structure. Since similar responses were required of the listeners in this and the earlier experiment, and the subjects were free to base their judgments on whatever features of the sequences that were salient to them, it may well be that those features perceived by novice adults are different from those perceived by the children in this study.

The developmental pattern was one in which the acquisition of the tonal hierarchy proceeded in an orderly manner, with the first distinction made being that between the scale and nonscale tones. Once the possible pitches of the tonal system were isolated, finer differentiation was made within the set of scale pitches. Although not logically necessary (for example, octave equivalence might have appeared earlier), a number of considerations suggest that this sequence of acquisition may reflect certain basic cognitive principles.

First, Dowling (1978) has noted that most musical cultures employ a relatively small number of pitches within an octave range. He, like Helmholtz (1863/1954), argued that discrete steps are desirable in order for melodic and rhythmic structure to be immediately apparent. Moreover, the number of scale steps within the octave falls within the range of elements on a single 
stimulus dimension that can be accurately encoded and remembered (Miller, 1956). Thus, the diatonicnondiatonic distinction limits the set of possible pitches to a subset that is small enough to be efficiently processed.

Second, in the history of Western music, the diatonic scale was originally used in many different modes, such that the tonic tone might be any one of the pitches of the scale. Only later did the major mode, and to a lesser extent, the minor mode, predominate over the other possible modes. Historically, then, the diatonic scale structure was prior to the emphasis given to certain patterned elements, such as the tonic triad chord, that are characteristic of the particular major and minor modes that have been most prevalent over the last 4 centuries. The notion that historical development should be paralled by its ontogenesis in children is a familiar one in other cognitive domains, such as language acquisition (Slobin, 1977).

Third, it might be argued that since musical relationships are defined only relative to other tones explicitly contained within the context (except perhaps for those few listeners with absolute pitch), it is essential to first develop an abstract internal framework that matches the most frequently appearing interval patterns of the scale. Once this framework is established, it may then be used to disambiguate the functions of the individual tones within the particular music context. Differentiation of the relative stabilities of tones within this system may then enable the listener to apprehend more subtle aspects of melodic and harmonic structure.

Finally, hierarchical structure itself confers certain advantages of economy on both processing and representation (cf. Simon, 1969). Hierarchical representations of knowledge structures (Keil, 1981) and procedures (Siegler, 1976) have been shown in other domains of cognitive development, and the notion of hierarchical differentiation has been influential for many years (Werner, 1948). Recently, Deutsch and Feroe (1981) provided a detailed model of the internal representation of tonal music that codes in an abstract, hierarchical form the complex time and pitch relationships of musical sequences. The present investigation characterizes the development of the internalized hierarchy of tonal functions, which appears to be significantly involved in interpreting the incoming acoustic signal during music perception.

Perhaps the single most impressive feature about the development of the hierarchy of stabilities is that it is implicit knowledge that must be extracted from experience with music in the tonal tradition. All children and most adults are unable to describe this knowledge. In a manner similar to natural language syntax, it develops without conscious awareness. In music, the structure is stable and tightly organized, and even young children are able to use surprisingly abstract distinctions to guide their intuitions about what are and are not well formed musical sequences.

\section{REFERENCES}

Bartlett, J. C., \& Dowlina, W. J. Recognition of transposed melodies: A key-distance effect in developmental perspective. Journal of Experimental Psychology: Human Perception and Performance, 1980, 6, 501-515.

Bruner, J. S., Olver, R. R., Greenfield, P. M., et al. Studies in cognitive growth. New York: Wiley, 1966.

Bharucha, J., \& Krumhansl, C. L. The representation of harmonic structure in music: Hierarchies of stability as a function of context. Cognition, in press.

Chana, H.-W., \& Trehub, S. Auditory processing of relational information by young infants. Journal of Experimental Child Psychology, 1977, 24, 324-331.

CHI, M. T. H. Knowledge structures and memory development. In R. S. Siegler (Ed.), Children's thinking: What develops? Hillsdale, N.J: Erlbaum, 1978.

Cole, M., \& Menss, B. Comparative studies of how people think. Cambridge, Mass: Harvard University Press, 1981.

Cundy, L. L., Cohen, A. J., \& Miller, J. Melody recognition: The experimental application of musical rules. Canadian Journal of Psychology, 1979, 33, 148-157.

Deutsch, D., \& Feroe, J. The internal representation of pitch structure in tonal music. Psychological Review, 1981, 88, 503522.

Dewar, K. M., Cuddy, L. L., \& Mewhort, D. J. K. Recognition memory for single tones with and without context. Journal of Experimental Psychology: Human Learning and Memory, 1977, 3, 60-67.

Dowling, W. J. Scale and contour: Two components of a theory of memory for melodies. Psychological Review, 1978, 85, 341354.

Dowling, W. J. The cognitive psychology of music. Humanities Association Review, 1979, 20, 58-67.

Dowling, W. J. Melodic information processing and its development. In D. Deutsch (Ed.), The psychology of music. New York: Academic Press, in press. (a)

Dowling, W. J. Musical and psychophysical scales: Their psychological reality. In T. Rice \& R. Falck (Eds.), Cross-cultural perspectives on music. Toronto: University of Toronto Press, in press. (b)

Dowling, W. J., \& Fujitani, D. S. Contour, interval, and pitch recognition in memory for melodies. Journal of the Acoustical Society of America, 1971, 49, 524-531.

Gardner, H., Davidson, L., \& McKernon, P. The acquisition of song: A developmental approach. In Proceedings of the National Symposium on the Teaching and Learning of Music. Reston, Va: Music Educators National Conference, 1980.

Gelman, R. Cognitive development. Annual Review of Psychology, 1978, 29, 297-332.

HzLmhoLTz, H. voN. [On the sensations of tones as a physiological basis for the theory of music] (A. J. Ellis, Ed. and trans.). New York: Dover, 1954. (Originally published, 1863.)

IMBERTY, M. L'acquisition des structures tonales chez l'enfant. Paris: Klincksieck, 1969.

Jefrries, T. B. Relationship of interval frequency count to ratings of melodic intervals. Journal of Experimental Psychology, 1974, 102, 903-905.

Johnson, S. C. Hierarchical clustering schemes. Psychometrika, 1967, 32, 361-372.

KEIL, F. C. Semantic and conceptual development: An ontological perspective. Cambridge, Mass: Harvard University Press, 1979.

KEIL, F. C. Constraints on knowledge and cognitive development. Psychological Review, 1981, 88, 197-227.

Kessen, W., Levine, J., \& Wendrich, K. A. The imitation of pitch in infants. Infant Behavior and Development, 1979, 2, 93-99.

Kosslyn, S. M., Pick, H. L., \& Fariello, G. R. Cognitive maps in children and men. Child Development, 1974, 45, 707-716. 
KrumhansL, C. L. The psychological representation of musical pitch in a tonal context. Cognitive Psychology, 1979, 11, 346374.

Krumhansl, C. L., Bharucha, J., \& Castellano, M. Key distance effects on perceived harmonic structure in music. Perception \& Psychophysics, in press.

Krumhansi, C. L., Bharucha, J., \& Kessler, E. J. Perceived harmonic structure of chords in three related musical keys. Journal of Experimental Psychology: Human Perception and Performance, 1982, 8, 24-36.

Krumhan8l, C. L., \& Kessler, E. J. Tracing the dynamic changes in perceived tonal organization in a spatial representation of musical keys. Psychological Review, 1982, 89, 334-368.

Krumhansi, C. L., \& Shepard, R. N. Quantification of the hierarchy of tonal functions within a diatonic context. Journal of Experimental Psychology: Human Perception and Performance, 1979, 5, 579-594.

Kruskal, J. B. Nonmetric multidimensional scaling: A numerical method. Psychometrika, 1964, 29, 28-42.

MANDLER, J. M. Structural invariants in development. In L. Liben (Ed.), Piaget and the foundations of knowledge. Hillsdale, N.J: Erlbaum, in press.

MANDleR, J. M., \& Defonest, M. Is there more than one way to recall a story? Child Development, $1979,50,886-889$.

Melson, W. H., \& MCCALL, R. B. Attentional responses of fivemonth girls to discrepant auditory stimuli. Child Development, 1970, 41, 1159-1171.

MEYER, L. B. Emotion and meaning in music. Chicago: University of Chicago Press, 1956.

Miller, G. A. The magic number seven, plus or minus two. Psychological Review, 1956, 63, 81-97.

Neisser, U. Cognition and reality. San Francisco: Freeman, 1976.
Nelson, K., \& Gruendel, J. Generalized event representations: Basic building blocks of cognitive development. In M. E. Lamb \& A. L. Brown (Eds.), Advances in developmental psychology (Vol. 1). Hillsdale, N.J: Erlbaum, 1981.

Ortmann, O. On the melodic relativity of tones. Psychological Monographs, 1926, 35(1, Whole No. 162).

Pick, A. D. Listening to melodies: Perceiving events. In A. D. Pick (Ed.), Perception and its development: A tribute to Eleanor J. Gibson. Hillsdale, N.J: Erlbaum, 1979.

Shepard, R. N. The analysis of proximities: Multidimensional scaling with an unknown distance function. I. Psychometrika, $1962,27,125-140$. (a)

ShE PARD, R. N. The analysis of proximities: Multidimensional scaling with an unknown distance function. II. Psychometrika, 1962, 27, 219-246. (b)

Siegler, R. S. Three aspects of cognitive development. Cognitive Psychology, 1976, 8, 481-520.

Simon, H. A. The sciences of the artificial. Cambridge, Mass: M.I.T. Press, 1969.

SLobin, D. I. Language change in childhood and in history. In J. MacNamara (Ed.), Language learning and thought. New York: Academic Press, 1977.

Vygotsky, L. A. [Thought and language] (E. Hanfmann \& G. Vakar, trans.). Cambridge, Mass: M.I.T. Press, 1962.

WERNER, H. Comparative psychology of mental development. New York: Universities Press, 1948.

Werner, H., \& Kaplan, B. Symbol formation. New York: Wiley, 1963.

Zenatti, A. Le développement génétique de la perception musicale. Monographies Françaises de Psychologie, 1969(Whole No. 17).

(Received for publication August 11, 1981; revision accepted February 10, 1982.) 